\title{
Theoretical and experimental investigations of the formation mechanisms of residual deformations of fibrous layered structure composites
}

\author{
Vitaly N. Paimushin ${ }^{1,2}$, Sergey A. Kholmogorov ${ }^{1}$, and Ildar B. Badriev,* \\ ${ }^{1}$ Kazan Tupolev National Research Technical University, 420111, 10 Karl Marx Str., Kazan, Russia \\ ${ }^{2}$ Kazan Federal University, 420008, 18 Kremlyovskaya Str., Kazan, Russia
}

\begin{abstract}
On the example of a unidirectional fibrous composite made of unidirectional carbon fibre composite and cold-hardening epoxy XT-118, tests of specimens with a stacking scheme $\left[+45^{0} /-45^{0}\right]_{2 s}$, where $\mathrm{s}$ is the number of monolayers, were carried out for cyclic loading by tension and compression. When the samples are stretched, both the fibers and the epoxy of composite are under shear stresses and stretching in the transverse direction by normal stresses, and under compression, under shear stresses and compression by normal stresses across the fibers. It is shown that in each loading cycle there is a consolidation of the material associated with the rearrangement of its microstructure, an increase in the secant modulus of elasticity and a decrease in the magnitude of the deformation increment at each cycle. The mechanism of accumulation of residual deformations under multiple loading of test samples was qualitatively studied and their level formed in the composite after each loading cycle was quantitatively found.
\end{abstract}

\section{Statement of the problem}

In the products of space technology for special purposes, nowadays, structural elements from fibrous composite materials $(\mathrm{CM})$, in particular, carbon plastic, are widely used. Such structural elements in the process of operation under multiple (multi-cycle) loading (in particular, thermal loading) should preserve the stability of geometric sizes and shapes. It was found that when ground-based testing of such products and during their operation, distortions of the measured parameters are always fixed, apparently due to the formation of residual deformations in the design. All the formation mechanisms of such residual deformations in fibrous composites in the process of exploitation of the structural elements made from them have not yet been fully revealed, which raises an urgent problem in the world science in the field of mechanics and machine building. We note that a numerical study of the geometrically and physically nonlinear problem of the three-point bending of test samples from layered fibrous composites having a rectangular cross-section was carried out in [1]. The problems of the bending of sandwich plates with a transversally soft core are discussed in [2-6].

\section{Experiments on cyclic deformation}

An extensive scientific literature [7-12], etc is devoted to the study of nonlinear deformation under cyclic loading of unidirectional fibrous composites. In particular, in work [12] such a study is carried out for a unidirectional composite on the basis of glass fibers under shearing conditions and repeated cyclic loading of specimens with a stacking scheme $\left[+45^{0} /-45^{0}\right]_{2 s}(s$ is the number of monolayers). On the basis of the obtained experimental data, the secant shear modulus $G_{12}$ is calculated and a conclusion is made about its fall after loading the sample with ten cycles. The authors explain the fall of the shear modulus by the degradation of the composite under conditions of shear deformations forming in the composite.

The carried out studies on the shear of test samples from fibrous composites with a polymer matrix show that the dependences between the tangential stresses and the corresponding shear deformations are essentially nonlinear, under conditions of loading and subsequent unloading, differ significantly among themselves, with residual deformations always being fixed on them. In particular, such studies of nonlinear deformation of a unidirectional fibrous composite on the basis of unidirectional carbon fibre composite and XT-118 epoxy under shearing conditions and repeated loading by tensile and compressive testing of specimnes with a stacking scheme $\left[+45^{0} /-45^{0}\right]_{2 s}$ were carried out. For tensile tests, specimens $\left[+45^{0} /-45^{0}\right]_{2 s} \quad(s=2)$ with an average thickness of $h=0.56 \mathrm{~mm}$, a width of $b=24.60$ $\mathrm{mm}$, and a working part length of $l=110 \mathrm{~mm}$ were used. For compression tests, the specimens were manufactured with an average thickness of $h=4.126 \mathrm{~mm}$, a width of $b=24.91 \mathrm{~mm}$ and a length of the working part of $l=25$ $\mathrm{mm}$. The tests were carried out two weeks after the manufacture of the test samples using the technology

\footnotetext{
"Corresponding author: ildar.badriev1@mail.ru
} 
described in [13] and eight months after their manufacture, when the polymerization of the binder composite could be considered complete.

When the stack of layers with the above laying scheme is stretched, both the fibres and the epoxy in the orthotropic axes are stretched in the direction of the fibres and the shearing by tangential stresses, and under compression, under compression and shearing by shearing stresses. The experiment was carried out under conditions of the same maximum normal stresses $\sigma_{x}$ in each "load-unload" cycle. This type of loading is sufficiently accurately equivalent to the work of a composite in a real construction, where the stresses should not exceed a certain specified (operational) value. At the previously found value of the ultimate (failure) stress $\sigma_{x}^{B}$ in the case of tensile tests, the specimens were brought to stresses $\sigma_{x}^{\max }=0$, the magnitude of which was $\sigma_{x}^{\max }=65 \mathrm{MPa}$, by repeated loading. Measuring of axial deformations was carried out with the help of a contact extensometer with a base of measurement $50 \mathrm{~mm}$. The deformation diagrams obtained under conditions of tension with the cycles "load-unloading" with the speed of the crosshead movement of $0.5 \mathrm{~mm} / \mathrm{min}$ are shown in Fig. 1 for samples [ $+45^{\%} /-$ $\left.45^{0}\right]_{2 s}, s=2$, that were kept eight months after their manufacture, and for the same specimens held for two weeks after manufacturing, are shown in Fig. 2.

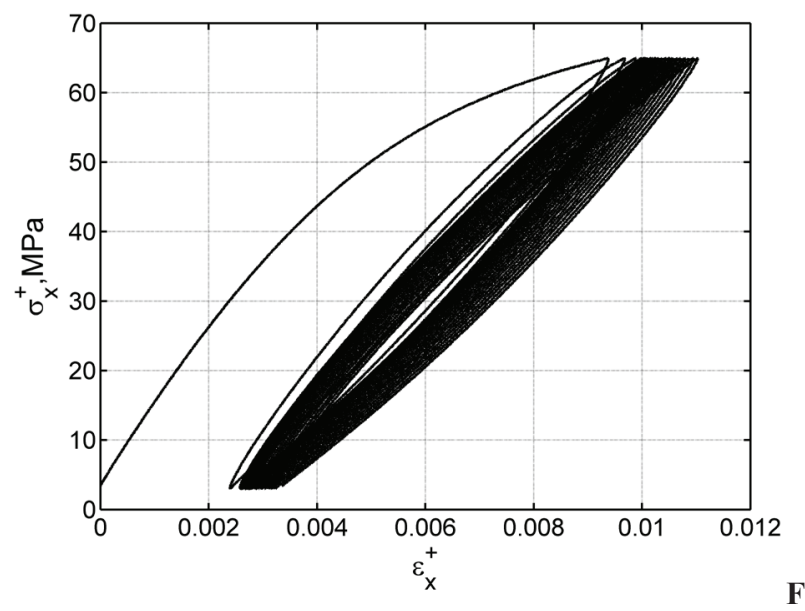

Fig. 1. Cyclic stretching diagram $\left(\sigma_{x}^{\max }=65 \mathrm{MPa}\right.$, exposure for eight months).

Comparing the results, it can be seen that holding the specimens at room temperature for eight months led to an increase in their rigidity by almost half. The loading and unloading branches do not coincide, forming a loop of hysteresis, and the axial strain $\varepsilon_{x}$ on each subsequent cycle with the same $\sigma_{x}$ in the cycle increases by a value of $\Delta \varepsilon_{x}^{(k)}$ (Fig. 1), where $k$ is the cycle number. As the number of cycles $\Delta \varepsilon_{x}^{(k)}$ increases, this indicates the process of hardening the fibrous composite at each loading cycle. Such a process is called the process of adaptability of a composite material. It should be pointed out that for a given $\sigma_{x}^{\max }<\sigma_{x}^{B}$ there is a limit number of cycles $k=N$ at which $\Delta \varepsilon_{x}^{(N)} \rightarrow \Delta \varepsilon_{x}$. Magnitude of $\Delta \varepsilon_{x}$ doesn't depend of cycle's number, but depends of loading rate.

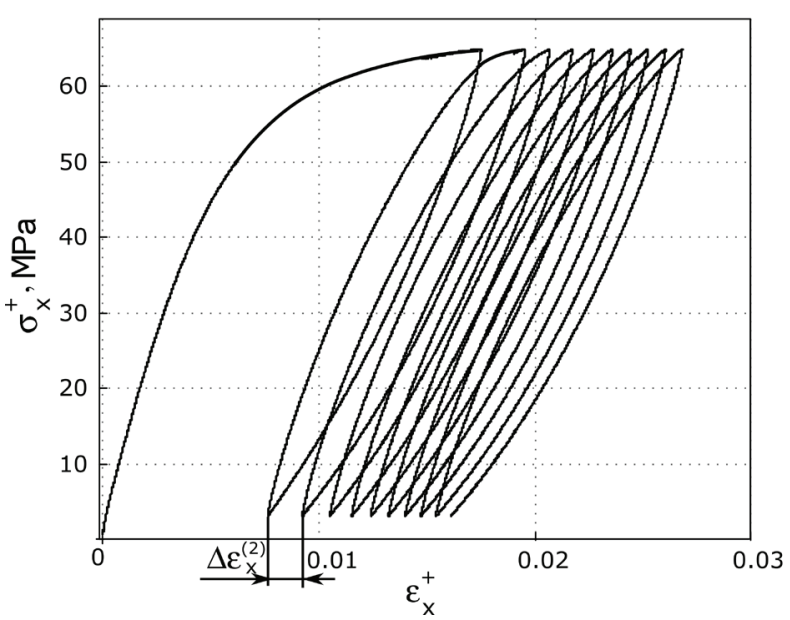

Fig. 2. Cyclic stretching diagram $\left(\sigma_{x}^{\max }=65 \mathrm{MPa}\right.$, exposure for two months).

The deformation diagram $\sigma_{x}^{+}=\sigma_{x}^{+}\left(\varepsilon_{x}\right)$ for the first two loading-unloading cycles for clarity on modified scales is shown in Fig. 3 with the limit points $A_{1}, A_{2}$ corresponding to $\sigma_{x}^{\max }=65 \mathrm{MPa}$, and $B_{1}, B_{2}$ corresponding to $\sigma_{x}^{\max }=4 \mathrm{MPa}$. These diagrams have the form of straight lines passing through the points $0 A_{1}, A_{1} B_{1}, B_{1} A_{2}, A_{2} B_{2}$.

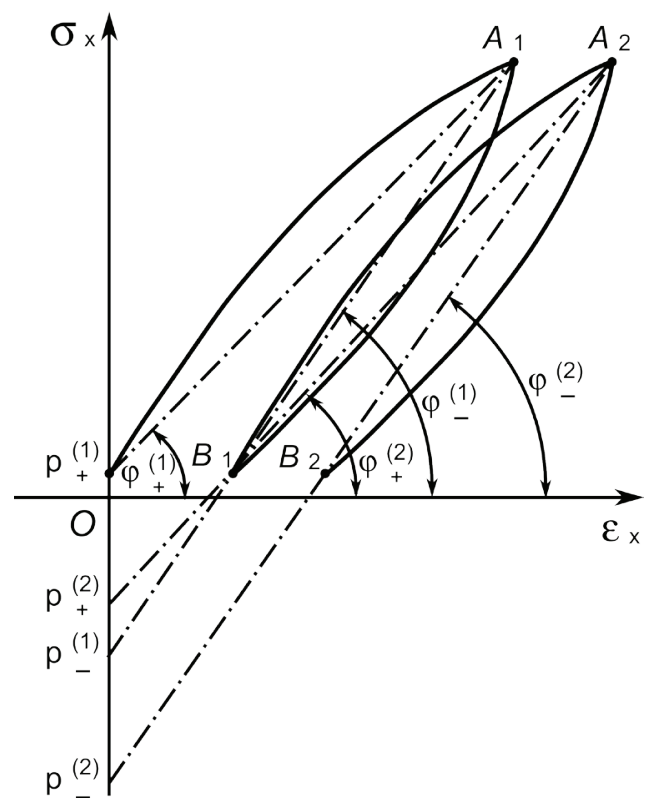

Fig. 3. Cyclic deformation scheme $\left(\sigma_{x}^{+}=\sigma_{x}^{+}\left(\varepsilon_{x}\right)\right)$.

The angles between these lines and the axis $0 \varepsilon_{x}$ are also shown, and the table 1 shows the values 
$E_{+}^{(i)}=\operatorname{tg} \varphi_{+}^{(i)}, \quad E_{-}^{(i)}=\operatorname{tg} \varphi_{-}^{(i)}$ representing the secant modulus of elasticity at the $i$-th load cycle (with the index "+") and unloading (with the "-" index). In addition, the values $p_{+}^{(i)}, p_{-}^{(i)}$ indicating coordinates of intersection points of the straight lines $A_{i} B_{i}, B_{i} A_{i+1}$ with the axis $0 \sigma_{x}^{+}$are given. The changes of values $\Delta \varepsilon_{x}^{(i)}$ on the first ten loading cycles can be traced through the table, and the dependence $\Delta \varepsilon_{x}^{(i)}=\Delta \varepsilon_{x}^{(i)}(i)$ is shown in Fig. 4. Analyzing the above results, it can be seen that even on the tenth cycle with the highest degree of accuracy the equalities $E_{+}^{(10)} \approx E_{-}^{(10)} \approx 8.31 \mathrm{GPa}$, $\Delta \varepsilon_{x}^{(10)} \approx 0, \quad p_{+}^{(10)} \approx p_{-}^{(10)} \approx-18.5 \mathrm{MPa}$ are fulfilled. Results similar to those described above were also obtained at $\sigma_{x}^{\max }=55 \mathrm{MPa}$ and $\sigma_{x}^{\max }=45 \mathrm{MPa}$. It has been established that as the reduction decreases of $\sigma_{x}^{\max }=65 \mathrm{MPa}$, a smoother decrease in values of $\Delta \varepsilon_{x}^{(i)}$ is observed, as well as a slight increase of the parameters $E_{+}^{(i)}, E_{-}^{(i)}, p_{-}^{(i)}$.

Table 1. The results of the tests for the multicyclic stretching.

\begin{tabular}{|c|c|c|c|c|c|}
\hline$i$ & $\begin{array}{c}E_{+}^{(i)} \\
\mathrm{GPa}\end{array}$ & $\begin{array}{c}E_{-}^{(i)} \\
\mathrm{GPa}\end{array}$ & $\Delta \varepsilon_{x}^{(i)} \cdot 10^{3}$ & $\begin{array}{c}p_{+}^{(i)}, \\
\mathrm{MPa}\end{array}$ & $\begin{array}{c}p_{-}^{(i)}, \\
\mathrm{MPa}\end{array}$ \\
\hline 1 & 6.59 & 8.9 & 2.39 & 3.45 & -18.14 \\
\hline 2 & 8.48 & 8.84 & 0.29 & -17.12 & -20.64 \\
\hline 3 & 8.59 & 8.69 & 0.09 & -19.98 & -20.91 \\
\hline 4 & 8.6 & 8.63 & 0.01 & -20.67 & -20.95 \\
\hline 5 & 8.56 & 8.51 & -0.03 & -20.75 & -20.32 \\
\hline 6 & $8, .9$ & 8.47 & -0.01 & -20.26 & -20.08 \\
\hline 7 & 8.47 & 8.44 & -0.004 & -20.08 & -19.72 \\
\hline 8 & 8.45 & 8.4 & -0.004 & -19.74 & -19.27 \\
\hline 9 & 8.42 & 8.34 & -0.005 & -19.31 & -18.6 \\
\hline 10 & 8.31 & 8.31 & -0.001 & -18.52 & -18.53 \\
\hline
\end{tabular}

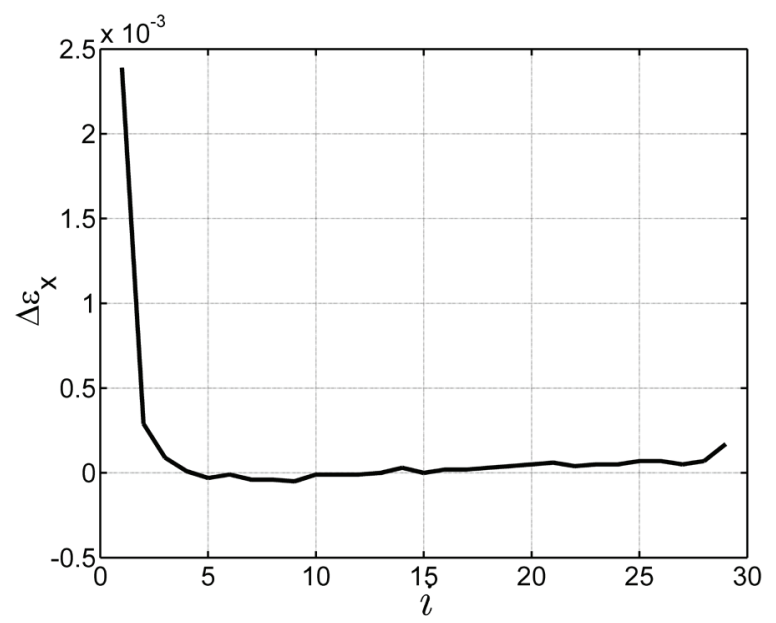

Fig. 4. Deformation increments at each loading cycle $\left(\sigma_{x}^{\max }=65 \mathrm{MPa}\right)$

Figure 5 shows the deformation diagram for compression tests of specimens held for two weeks after their manufacture. The speed of traversing the machine in the experiment was $0.5 \mathrm{~mm} / \mathrm{min}$. It should be noted that the axial strain $\varepsilon_{x}^{-} \approx 0.02$ at the first loading cycle corresponds to a higher value of the normal stresses $\sigma_{x}^{\max }=-80 \mathrm{MPa}$, which is $19 \%$ higher than $\sigma_{x}^{\max }=65 \mathrm{MPa}$ for the same $\varepsilon_{x}^{-} \approx 0.02$. The main regularities of the curve $\sigma_{x}^{-}=\sigma_{x}^{-}\left(\varepsilon_{x}^{-}\right)$(Fig. 2) remained similar to the curve $\sigma_{x}^{+}=\sigma_{x}^{+}\left(\varepsilon_{x}^{+}\right)$(Fig. 5).

In order to determine the tangential shear modulus as a function $G_{12}^{(k)}=G_{12}^{(k)}\left(\gamma_{12}\right)$, where $\gamma_{12}$ is shearing strain, and to study its variation in each loading cycle of the tested specimens of the diagrams $\sigma_{x}^{+}=\sigma_{x}^{+}\left(\varepsilon_{x}^{+}\right)$and $\sigma_{x}^{-}=\sigma_{x}^{-}\left(\varepsilon_{x}^{-}\right)$under tension (Fig. 2) and compression (Fig. 5), they were processed in accordance with the technique proposed in [14].

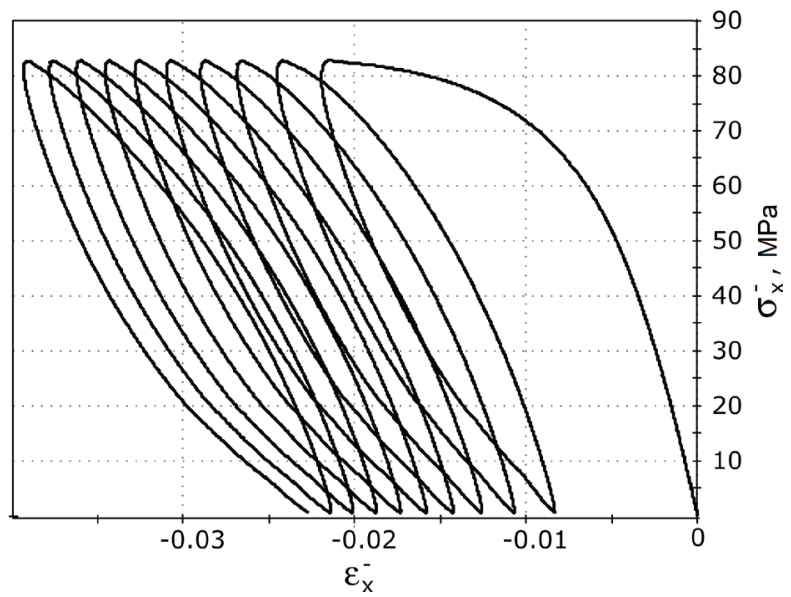

Fig. 5. Cyclic compression diagram $\left(\sigma_{x}^{\max }=-80 \mathrm{MPa}\right.$, exposure for eight months).

The dependencies $G_{12}^{(k)}=G_{12}^{(k)}\left(\gamma_{12}\right)$ for the first and the tenth loading cycles (both in tension and compression) are shown in Fig. 6. In this figure, the line $-\square$. corresponds to the first cycle under tension, $-\boldsymbol{\Delta}-\square \square$ to the first cycle under compression, $--\cdot--\square$ to the tenth cycle under tension and $--\mathbf{\Delta}--$ to $\square$ the tenth cycle upon compression.

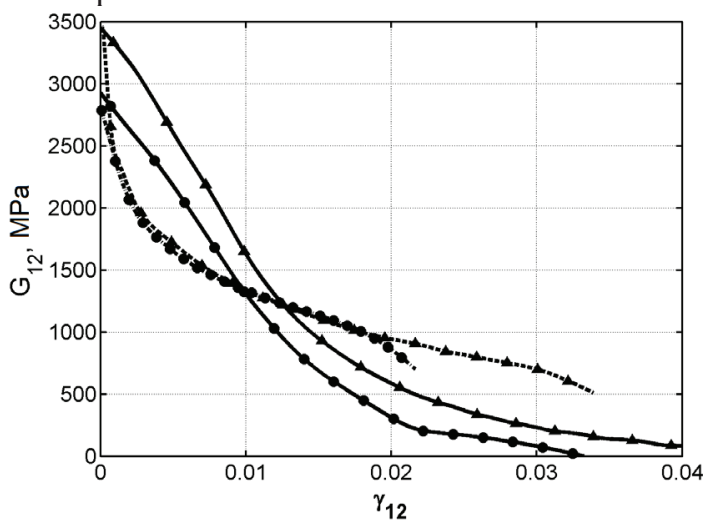

Fig. 6. Dependencies $G_{12}^{(k)}=G_{12}^{(k)}\left(\gamma_{12}\right)$ for the first and the tenth loading cycles. 
It can be seen that the curve $G_{12}^{(k)-}=G_{12}^{(k)-}\left(\gamma_{12}\right)$ lies above the curve $G_{12}^{(k)+}=G_{12}^{(k)+}\left(\gamma_{12}\right)$ over the entire range. The value of $G_{12}^{(1)-}$ at $\gamma_{12}=0$ is $3400 \mathrm{MPa}$, and value of $G_{12}^{(1)+} \square$ is $2750 \mathrm{M} \mathrm{Pa}$. This difference between the two curves remains practically constant throughout the entire range of variation $\gamma_{12}$. The curves $G_{12}^{(10)-}$ and $G_{12}^{(10)+}$ practically coincide on all the range of $\gamma_{12}$ presented in the tests, and on the sector $0<\gamma_{12}<0.01$ they are below the curves $G_{12}^{(1)-}$ and $G_{12}^{(1)+}$, and in the section $\gamma_{12}>0.01 \square$ they are above the curves $G_{12}^{(1)-}$ and $G_{12}^{(1)+}$. Curves $G_{12}^{(10)-}, G_{12}^{(10)+}, G_{12}^{(1)-}$ and $G_{12}^{(1)+}$ intersect in a neighborhood $\gamma_{12} \approx 0.01$. The diagrams for tension and compression for the first and subsequent cycles are non-linear over the entire range of variation. The diagrams for tension and compression for the first and subsequent cycles are non-linear the whole range of change $\gamma_{12}$.

\section{Conclusion}

In contrast to the effects known for loading multilayer composites in a direction transverse to the fibers and describing their models (developed by V.V. Vasilyev and then by A.N. Alfutov and co-authors), under repeated loading and unloading by tangential stresses on each cycle, a change of deformation diagrams is observed. The effect has been established experimentally in that there is such a limiting amplitude value of the stresses formed during testing, to which the parameters of the deformation diagrams stabilize, when exceeded, the hysteresis loop widens up to failure. The loss of structural strength is caused either by the destruction of elements in a sufficiently large area, or by the development of cracks. In fibrous composites, their failure is caused, in particular, by loss of stability or reinforcing fibers at the micro-level, or a fiber bundle (monolayer) at the meso-level, or the entire structural element at the macro-level, often accompanied by stratification of the composite structure. This effect, as a rule, is visually observed in experiments both in the compression of test samples and in their three-point bending. These types of tests are currently regulated by both Russian and international standards. The process of destruction caused by the described effects of the first two species, in the mechanics of composite materials and structures made of them, is practically not taken into account in modeling and analysis of their strength. Therefore, the actual problem is the creation of refined models of deformation and destruction of multilayer composites, taking into account the possibility of realizing non classical buckling of fibers at the microlevel (in scales of the cross-sectional dimension of the fiber), a fiber bundle at the meso-level (in the scale of one monolayer), taking into account the interaction of fibers and a fiber bundle with their surrounding matrix, as well as shear and flexural-shear buckling at the macro-level (in the scale of the thickness of structural elements in the form of a plates and shells and crosssectional dimensions of structural elements in the form of rods), theoretical modeling of tests of specimens from fibrous composites for single and multiple stretching, compression, stretching-compression and three-point bending with appropriate experimental studies, as well as significant refinement of fiber composite testing standards with experimental confirmation of the results of theoretical studies. All these studies are necessary to show that in the process of deformation of structural elements from laminated fibrous composites in monolayers in which a shear stressed-deformed state is formed, structural changes occur in the composite both under loading and unloading of the structure, leading to the formation of Residual deformations. It is also necessary to identify and theoretically describe the mechanism for the formation of such structural changes.

The results of the research are obtained within the framework of the state task of the Ministry of Education and Science of Russia No. 9.5762.2017, project No. 9.1395.2017 and due to the grant of the Russian Scientific Foundation (project No. 1611-10299).

\section{References}

1. I.B. Badriev, M.V. Makarov, V.N. Paimushin, Mathematical Simulation of Nonlinear Problem of Three-point Composite Sample Bending Test, Procedia Engineering, 150, 1056-1062 (2016)

2. I.B. Badriev, M.V. Makarov, V.N. Paimushin, Numerical Investigation of Physically Nonlinear Problem of Sandwich Plate Bending, Procedia Engineering, 150, 1050-1055 (2016)

3. I.B. Badriev, M.V. Makarov, V.N. Paimushin, Solvability of a physically and geometrically nonlinear problem of the theory of sandwich plates with transversal-soft core, Russian Mathematics, 59, 10, 57-60 (2015)

4. I.B. Badriev, M.V. Makarov, V.N. Paimushin, Contact statement of mechanical problems of reinforced on a contour sandwich plates with transversally-soft core, Russian Mathematics, 61, 1, 69-75 (2017)

5. I.B. Badriev, G.Z. Garipova, M.V. Makarov, V.N. Paimushin, R.F. Khabibullin, Solving physically nonlinear equilibrium problems for sandwich plates with a transversally soft core, Lobachevskii Journal of Mathematics, 36, 4, 474481 (2015)

6. I.B. Badriev, M.V. Makarov, V.N. Paimushin, On the interaction of composite plate having a vibration-absorbing covering with incident acoustic wave, , Russian Mathematics, 59, 3, 66-71 (2015)

7. S. Ogihara, S. Kobayashi, K.L. Reifsnider, Characterization of nonlinear behavior of carbon/epoxy unidirectional and angleply laminates, 
Advanced Composite Materials: The Official Journal of the Japan Society of Composite Materials, 11, 3, 239-254 (2003)

8. T.A. Bogetti, C.P.R. Hoppel, V.M. Harik, J.F. Newill, B.P. Burns, Predicting the nonlinear response and progressive failure of composite laminates, Composites Science and Technology, 64, 3-4, 329-342 (2004)

9. M. Kashtalyan, C. Soutis, Stiffnes degradation in cross-ply laminates damaged by transverse cracking and splitting, Composites: Part A, 31, 335-351 (2000)

10. A.M. Dumansky, L.P. Tairova, The prediction of viscoelastic properties of layered composites on example of cross ply carbon reinforced plastic, World Congress on Engineering, II, London, UK 24 July, 2007, 1346-1351 (2007)

11. W.Van Paepegem, I. De Baere, J. Degrieck, Modelling the nonlinear shear stress-strain response of glass fiber-reinforced composites. Part I: Experimental results, Composite Science and Technology, 66, 1455-1464 (2006).

12. A.M. Dumansky, L.P. Tairova, I. Gorlach, M.A. Alimov, A design-experiment study of nonlinear properties of coal-plastic, Journal of Machinery Manufacture and Reliability, 40, 5, 483488 (2011).

13. R.A. Kayumov, S.A. Lukankin, V.N. Paimushin, S.A. Kholmogorov, Identification of mechanical properties of fiber-reinforced composites, Uchenye Zapiski Kazanskogo Universiteta. Seriya FizikoMatematicheskie Nauki, 157, 4, 112-132 (2015).

14. V.N. Paimushin, D.V. Tarlakovskii, S.A. Kholmogorov, On non-classical buckling mode and failure of composite laminated specimens under the three-point bending, Uchenye Zapiski Kazanskogo Universiteta. Seriya FizikoMatematicheskie Nauki, 158, 3, 350-375 (2015). 